\title{
Heater Component Modal Analysis of Electronic Gun under High Temperature Condition
}

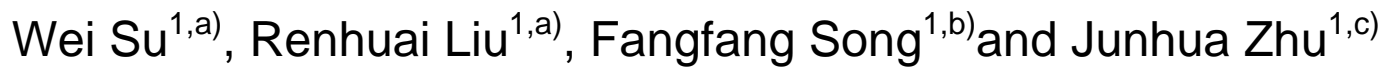 \\ ${ }^{1}$ Scienceand Technology on Reliability Physics and Application Technology of Electronic \\ Component Laboratory, China Electronic Product Reliability and Environmental Testing Research \\ Institute, Guangzhou 510610,China. \\ a)suwei5@126.com, ${ }^{\text {b) }}$ songff@ceprei.com, ${ }^{\mathrm{c}}$ jhzhu99@163.com
}

Keywords: Heater component; Modal; Electric gun.

\begin{abstract}
This paper calculates thermal-structural coupling analysis of the heater component considering thermal radiation and heat conduction. We get the heater components steady temperature field and stress distribution. The thermalstress is taken as prestressed force and finite element analysis is used to obtain vibration models. The corresponding modal frequency and modal vibration mode areobtained by simulation analysis and the results provide beneficial references for solving the problem of anti vibration reliability under high temperature.
\end{abstract}

\section{INTRODUCTION}

As the heart of weapon, Traveling Wave Tubes (TWT) is widely used in radar, navigation and communication systems. In order to improve the performance and quality of TWT, many scholars and research in stitutions do their research about the status and progress of TWT reliability of thermal design, vibration reliability. Electron gun is the key component of TWT, and heater component in electron gun is prone to failure under the condition of high temperature and vibration. The related research on this question has not yet been underway.

Electron gun heated by hot wire in normal work makes cathode temperature about $1000^{\circ} \mathrm{C}$, hot wire working temperature about $1100^{\circ} \mathrm{C}[1-2]$.The high temperature, produced during electronic gun working, results to the thermal-stress of the components, and has effects to the parameters and lifetime of the gun. Electron gun often sustains the effect of mechanical shock and vibration, and heater components will break under the effect of temperature and vibration [3]. According to the statistics, heater component fracture occupies more than sixty percent of electron gun failure.

\section{STEADY THERMAL STRESS ANALYSYS}

The high temperature, produced during electronic gun working, results to the thermal-stress of the components, and has effects to the parameters and life time of the gun[4]. As the key component of TWT, electron gun is one of the components bearing the most complicate loads and easiest to fail. Overall modeling of gun adopts entity model, with the exception of heater by adopting tetrahedron unit, and the rest of the entity adopts hexahedral grid. Solid model finite element model of electron gun is shown in Fig.1(a,b). The heat generation rate defined as body load is applied to heater finite element and simulates current heating[3]. Convection boundary conditions are imposed on shell of the gun. Heat transference in the interior of the electron gun is mainly by radiation and heat conduction because the internal is vacuum. Cathode is heated by heater radiation [5], therefore, the inner surface of cathode drum, heater surface and bottom of cathode is defined as a radiation group [6]. 


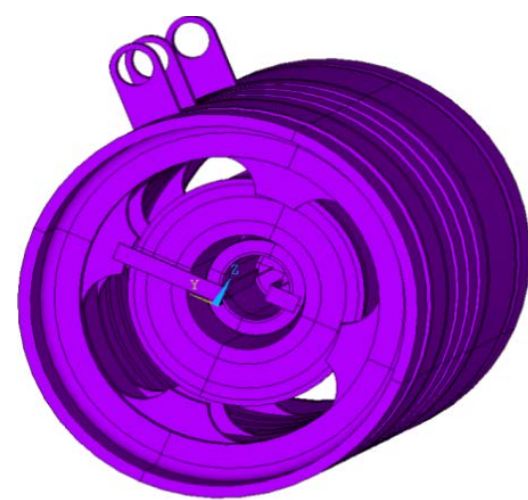

a. Solid model

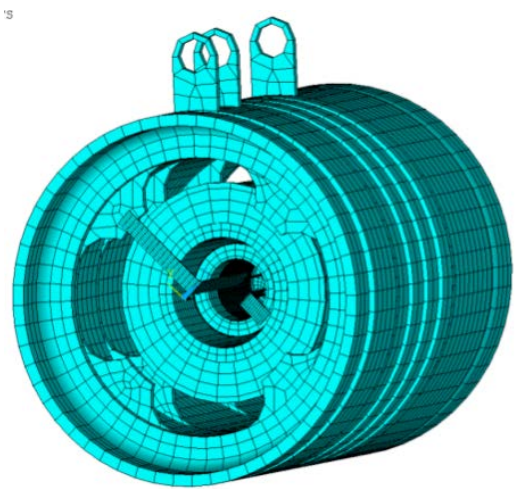

b. Finite element model

FIGURE 1.Model of electron gun

Thermal balance equation Eq.(1) shows that the required heat of heating up object should be balanced against heat introduced into body and heat generated within object.

$$
\rho c \frac{\partial T}{\partial t}-\frac{\partial}{\partial x}\left[\kappa_{x} \frac{\partial T}{\partial x}\right]-\frac{\partial}{\partial y}\left[\kappa_{y} \frac{\partial T}{\partial y}\right]-\frac{\partial}{\partial z}\left[\kappa_{z} \frac{\partial T}{\partial z}\right]-\rho Q=0
$$

In the equation, $T(x, y, z, t)$ expresses temperature field, $\rho$ expresses material density, $c$ expresses failure rate function, $t$ expresses time, $k_{x}, k_{y}, k_{z}$ expresses coefficient of heat conduction, $Q$ expresses heat source density.

Before the calculation between planar radiation heat energy, the shape factor $F_{i j}$ should be considered, and Radiant energy which is shown in Fig.2 is applied from a plane $i$ to another plan $\mathrm{j}$, $A_{i}, A_{j}$ expresses area of plane.

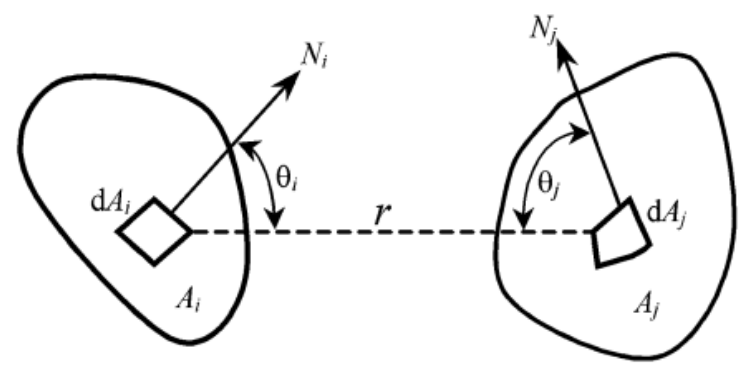

Figure2.Heat radiation between two planes

Then Eq.(2) can be obtained as follows:

$$
F_{i j}=\int_{A_{i}} \int_{A_{j}} \frac{\cos \theta_{i} \cos \theta_{j}}{\pi r^{2}} d\left(A_{i}\right) d\left(A_{j}\right)
$$

Cathode and the outer surface of cathode, heat shield tube and the inner surface of controller are defined as another radiation groups. Other component temperature is low, and heat transfers mainly by heat conduction, so their thermal radiations can be ignored. Thermal analysis entity unit SOLID90 is used in the steady state of heat conduction Temperature field distribution of electron gun is showed in Fig.3(a). Base on temperature field results, thermal stress

is also calculated by changing thermal elements into structural elements. Structural stress finite element cloud of electron gun are showed in Fig.3(b). 


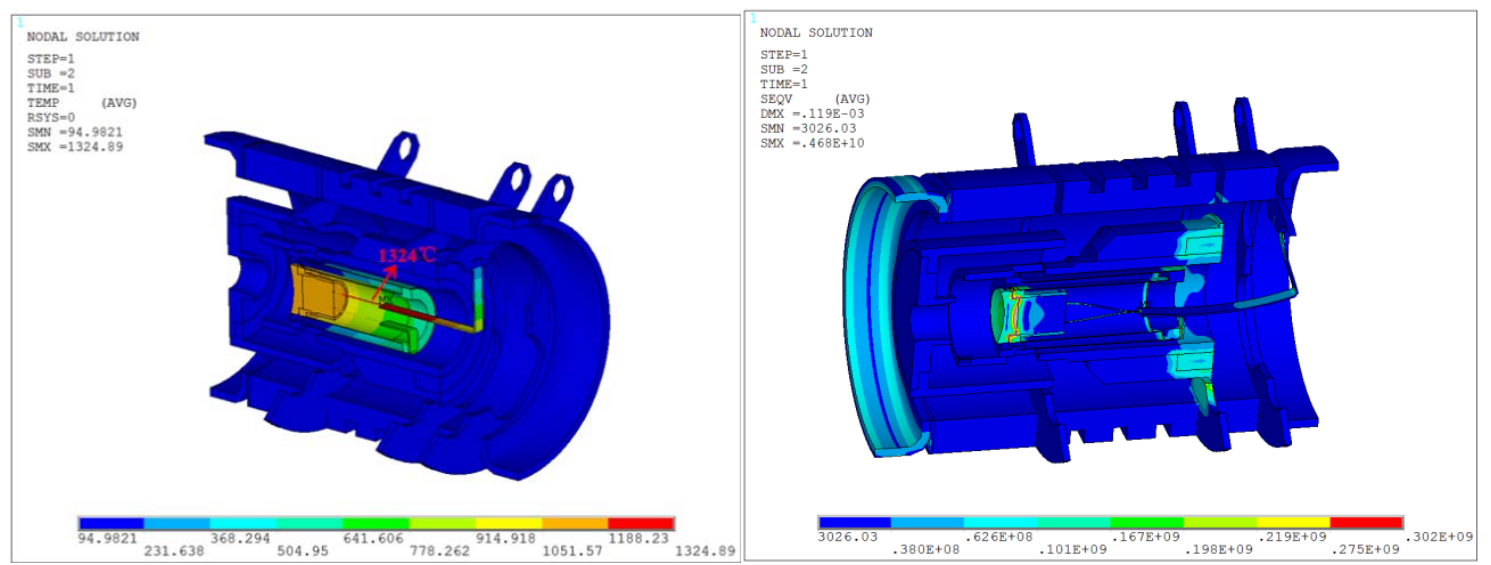

a. Distribution of steady temperature

b. Distribution of thermal stress

FIGURE3.Distribution of temperature and thermal stress

\section{MODALANALYSIS}

The thermalstress is taken as prestressed force, then, natural frequencies and vibration mode can be obtained by modal analysis. The structure of the whole dynamic balance equation can be expressed as Eq.(3).

$$
[M]\{\ddot{u}\}+[C]\{\dot{u}\}+[K]\{u\}=0
$$

In the equation, $[M]$ expresses mass matrix, $[C]$ expresses damp matrix, $[K]$ expresses stiffness matrix, $\{\ddot{u}\}$ expresses note acceleration vector, $\{\dot{u}\}$ expresses note velocity vector, $\{u\}$ expresses note displacement vector. In order to keep precision and speed of calculation, modal calculation adopts lanczos method and invokes sparse matrix equation solver. Because concerned acceleration power spectrum ranges from $10 \mathrm{~Hz}$ to $2000 \mathrm{~Hz}$, so we only need to calculate and expand modal in this range. The first modal vibration mode and the second modal vibration mode are showed in Fig.4(a,b).

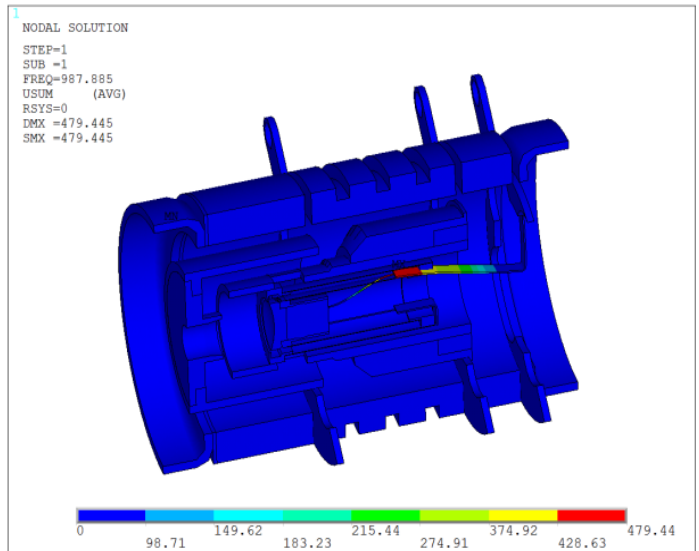

a. The first modal vibration mode

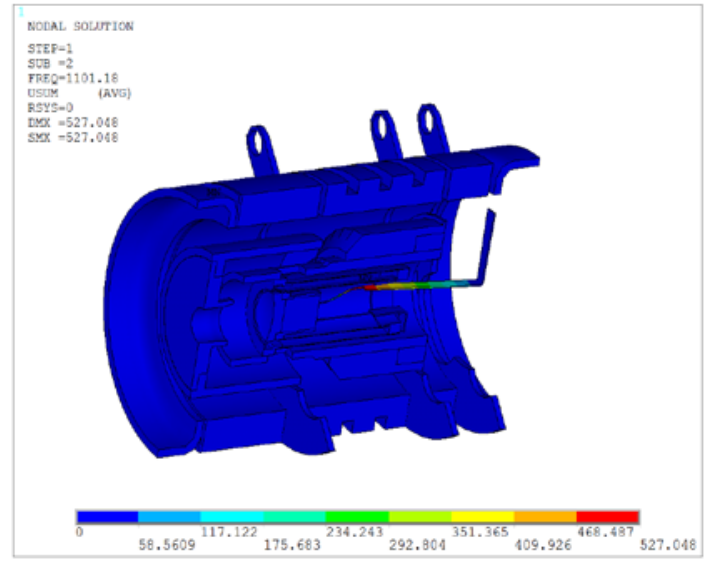

b. The second modal vibration mode

FIGURE 4.Modal vibration mode

\section{CONCLUSION}

First, this paper calculates thermal-structural coupling analysis of electron gun by use of ANSYS, and the result Fig.3(a) show that the temperature of the heater component after reaching steady state is about $1324^{\circ} \mathrm{C}$, the cathode temperature is about $1067^{\circ} \mathrm{C}$. This is similar to the test data, and the difference is mainly due to the actual welding situation in the internal components of the gun which cannot fully reflect in the simulation. Fig.3(b) show that the stress mainly concentrates in the heater terminal of cathode ceramic and the junction between platinum piece and heater .The stress value is about 302MPa.

Second , through putting thermal stress as prestressed force, natural frequencies and vibration 
modes can be ob-

tained by modal analysis. Fig.4 shows that the first natural frequency of heater component is about $479 \mathrm{~Hz}$ and second modal vibration mode is about $527 \mathrm{~Hz}$. The max mode of vibration amplitude occurs the junction between platinum piece and heater, and the junction is the most dangerous place prone to breakage. The results provide beneficial references for solving the problem of anti vibration reliability under high temperature.

\section{ACKNOWLEDGMENTS}

This work was financially supported by the Distinguished Young Scientist Program of Guangdong Province (2015A030306002),National Natural Science Foundation of China (51505089), Natural Science Foundation of Guangdong Province (2016A030313672) and Pearl River S\&T Nova Program of Guangzhou (2014J2200086).

\section{REFERENCES}

[1] Xijin Tian, Olgierd A Palusinski. Reliability, thermal analysis and optimization design of multilayer PCB boards [A] . Proceedings Annual Reliability and Maintainability Symposium [C].2002.392-398.

[2] Wei Su, Junhua Zhu, Renhuai Liu, Fangfang Song Thermal-structural Coupling Analysis of Electron Gun in Air-borne TWT,ICRMS,2014.662-665.

[3] Sharma R K, Sinha A K, Gupta R K. Thermal Analysis Gun for a Miniature Helix TWT[J] . IETE Technical Review, 2000,17(5):269-274.

[4] Baek S W, Lee J H , Cho K H , et al . Thermal Analysis of Output Connector for Traveling Wave Tube [C] .Third IEEE International Vacuum Electronics Conference, USA, 2002:230-231.

[5] Kim H S, Uhm H S, Baek S W. Thermal and Structural Analysis on Output Coupler of Helix TWT [C] . Inter -national Conference on Plasma Science, 2003: 174-177.

[6] Li B, Yang Z H, Li J Q, et al . Theory and Design of Microwave Simulator Suite[ J] . IEEE Trans Electron Devices, 2009,56(5): 919-927. 